\title{
Posición de impactación de terceros molares en adultos peruanos
}

\author{
Third molar impaction position in Peruvian adults
}

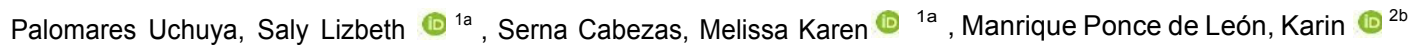

\begin{abstract}
Objetivo: Determinar la posición de los terceros molares en pacientes atendidos en un centro de diagnóstico por imágenes en Lima durante el período 2017 - 2020.Metodologia: Se realizó una investigación observacional, descriptivo, transversal y retrospectivo; el cual, estuvo conformado por una muestra de 253 radiografías panorámicas de pacientes atendidos en un centro radiológico privado. Se evaluó la posición de los terceros molares inferiores, la profundidad de impacto, y su posición de impactación en relación con la rama mandibular utilizando la clasificación de Pell y Gregory; así como, la clasificación de Winter. Los datos fueron analizados en el paquete estadístico SPSS vs.26. Resultado: Se observó que la posición vertical y mesioangular fueron las más comunes con un $14,6 \%$ y 13,2\%; respectivamente. El tipo I y II según Pell y Gregory, fue el más común con valores de 15,4\% y 14,4\%; así como, la clase B con 15,4\%.Conclusión: La posición de impactación vertical, mesioangular, tipo B y clase II fueron las más frecuentes para los terceros molares inferiores.
\end{abstract}

Palabras claves: Tercer molar; Diente impactado; Diente permanente; Radiografía panorámica. Fuente: (DeCS BIREME)

\section{ABSTRACT}

Objective. To determine the position of the third molars in patients treated in a diagnostic imaging center in Lima during the period 2017-2020. Methodology. An observational, descriptive, cross-sectional and retrospective research was carried out; which consisted of a sample of 253 panoramic radiographs of patients treated in a private radiological center. The position of the lower third molars, the depth of impact, and their impaction position in relation to the mandibular ramus were evaluated using the Pell and Gregory classification; as well as the Winter classification. The data were analyzed in the statistical package SPSS vs. 26 . Outcome. It was observed that the vertical and mesioangular position were the most common with $14.6 \%$ and $13.2 \%$; respectively. Type I and II according to Pell and Gregory was the most common with values of $15.4 \%$ and $14.4 \%$; as well as, class B with $15.4 \%$. Conclusion. The vertical and mesioangular impaction position, type B and class II were the most frequent for the lower third molars.

Keywords: Third molar; Impacted tooth, Permanent tooth; Panoramic radiograph. Source:( MeSH NLM)

Recibido: 15 de Mayo de 2021

Aprobado: 05 de Julio de 2021

Publicado: 08 de julio de 2021

${ }^{1}$ Universidad Inca Garcilaso de la Vega. Lima Perú

${ }^{2}$ Universidad Cesar Vallejo. Lima Perú

a Bachiller en Odontología

${ }^{\mathrm{b}}$ Docente

Este es un artículo de acceso abierto distribuido bajo la licencia Creative Commons Atribución 4.0 Internacional (CC BY 4.0)

Correspondencia:

https://creativecommons.org/licenses/by/4.0/deed.es

Saly Lizbet Palomares Uchuya

Dirección: Calle. Juana Larco de Dammert 154.Urb. Covima, La Molina. Lima.Perú

Correo electrónico: salypalomares27@gmail.com

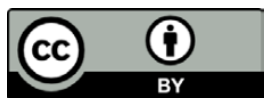

Citar como: Palomares Uchuya, S L, Serna Cabezas M K, Manrique Ponce de León, K. Posición de impactación de terceros molares en adultos peruanos. KIRU. 2021 Jul-Set; 18(3): 153- 159. https://doi.org/10.24265/kiru.2021.v18n3.04 


\section{INTRODUCCIÓN}

La impactación de los dientes permanentes tiene como resultado a la insuficiencia de erupción del diente o también la disposición de un obstáculo en el camino (1). Presentándose como una falla en el brote de la pieza por erupcionar, que puede ser el resultado de un obstáculo en la trayectoria de la posición inadecuada del diente ${ }^{(2)}$. Las causas de la impactación dentaria comprenden de factores sistémicos y locales. Entre los factores sistémicos se encuentran los hábitos nutricionales, y las deficiencias genéticas, mientras tanto, en los factores locales intervienen el tamaño y posición de los dientes continuos, como el hueso compacto que cubre al diente, el trayecto de erupción, y longitud inadecuada del arco dental (1). Los factores que afectan la impactación se encuentran en un rango de edad, que serán visualizadas en las características radiográficas, en su desarrollo del diente y en la etapa de la erupción dental ${ }^{(2)}$.

En la dentición humana el tercer molar mandibular impactado es el más común en cuanto su localización y posición en las arcadas dentarias una prevalencia aproximadamente de $73 \%$ en adultos jóvenes, y se establece que las mujeres tienen una mayor incidencia de impactación en comparación con los hombres ${ }^{(4)}$. Sin embargo, diversos estudios han obtenido niveles de prevalencia alrededor del $70 \%$, observándose que el alto grado de variación de este porcentaje obedece a diferencias étnicas y rasgos raciales, afectando diferenciadamente a cada tipo de población ${ }^{(5)}$. Además los terceros molares tiene variaciones morfológicas, tanto coronal como radicular en su formación ,en un $90 \%$ de los casos se evidencia que comienza a los 11 años de edad aproximadamente, y varia en su periodo de erupción entre los 18 a 20 años de edad ${ }^{(6)}$.

La anatomía y la posición de los terceros molares mandibulares tienen un factor local importante para evaluar la dificultad ante un procedimiento quirúrgico, considerando que es uno de los tratamientos odontológicos más comunes ${ }^{7}$. Los terceros molares tienden en aumentar el riesgo de complicaciones, como parestesia nerviosa, osteítis alveolar, hemorragia o incluso fractura de la mandíbula, también como una lesión del canal alveolar inferior asociado a una extracción, estas complicaciones se pueden anticipar usando radiografías, que pueden ayudar al cirujano. Por eso actualmente, la radiografía panorámica es la técnica más selectiva para evaluar los terceros molares mandibulares impactados ${ }^{(8)}$. Los terceros molares impactados pueden llevar a complicaciones como la pericoronaritis, pérdida de hueso periodontal, reabsorción radicular del diente impactado o del segundo molar adyacente, mayor riesgo de caries en el diente adyacente y de fractura en el ángulo mandibular. Con menor frecuencia podrían asociarse a quistes y tumores ${ }^{(3,9)}$.El conocimiento es clínicamente importante porque algunos de los patrones de terceros molares impactados se asocian con un mayor riesgo de caries en el diente adyacente, y fractura del ángulo mandibular.

Además, en el Perú no son muchos los estudios que han atendido esta problemática y viendo la amplia variedad de razas que existen en el país, se hace necesario disponer de evidencia que ayude a determinar el mejor tratamiento. En cuanto al contexto metodológico, esta investigación emplea el análisis de Winter y de Pell y Gregory con base en radiografías panorámicas de los pacientes, siendo técnicas que han sido validadas y desarrolladas en otros estudios lo que permite la discusión de los resultados. Asimismo, el estudio se justifica desde lo práctico porque aporta al centro de diagnóstico, información respecto a la posición, tipo y clase de los terceros molares más prevalentes en los pacientes, favoreciendo en el diagnóstico y tratamiento que se aplicarán y disminuyendo el riesgo de presentarse complicaciones postoperatorias. Por ello, el propósito de este estudio fue evaluar la posición de los terceros molares en pacientes atendidos en un centro de diagnóstico por imágenes en Lima durante el período 2017 - 2020.

\section{METODOLOGIA}

Estudio descriptivo ${ }^{(10,11)}$. La población estuvo conformada por 3500 radiografías panorámicas de pacientes atendidos en un Centro de Diagnóstico por Imágenes, Lima del periodo20172020. La muestra se obtuvo utilizando una fórmula para estimar una proporción, obteniéndose 253 radiografías panorámicas de pacientes con edades entre 18 a 58 años de edad y de ambos géneros, las cuales fueron 
seleccionados por criterios de inclusión, como nitidez y que por lo menos presenten un tercer molar acompañado de un segundo molar para cumplir con el estudio, se excluyeron radiografías con anomalías maxilofaciales y antecedentes de traumatismo en la región maxilofacial. El muestreo fue de tipo probabilístico aleatorio. Se solicitó el permiso al comité de investigación y centro radiológico privado, para el acceso a su registro digital de radiografías. Se utilizó una ficha de recolección de datos la cual estuvo conformada por ítems como género, edad del paciente, patrón de la impactación en función a la profundidad, posición referente a la rama mandibular y el ángulo de impactación con respecto al plano oclusal, según la clasificación de Pell y Gregory y Winter.

Para dicho análisis, intervinieron dos examinadores (el investigador y un especialista en radiología oral). Posteriormente, se procedió a obtener el índice de concordancia Kappa para evaluar la correlación interexaminador. En el proceso de calibración, se obtuvo un coeficiente de Kappa de 0.857 para la pieza 38 y 48 Inter observador; dado los resultados obtenidos, este instrumento es válido y confiable. Se recolectaron los datos en una ficha donde la profundidad de impactación, la cual se define como la relación de la unión amelocementaria del tercer molar en relación con la cresta ósea, se clasificó: A como la unión amelocementaria por encima de la cresta ósea; B que parte desde la unión amelocementaria debajo de la cresta ósea; y C, en la unión amelocementaria completo debajo de la cresta del hueso.

La posición referente a la rama mandibular se determinó como la posición de la superficie distal de la corona del tercer molar en relación con el borde anterior de la rama ascendente utilizando la clasificación de Pell y Gregory: Clase I, la superficie distal del molar esta, adelante del borde anterior de la rama ,la rama no cubre la corona, Clase II, la superficie distal del molar posterior al borde anterior de la rama parte de la corona cubierta por la rama, Clase III, la superficie distal del molar posterior al borde anterior de la rama, corona completa cubierta por la rama.

El ángulo de impactación se define como el ángulo entre los ejes longitudinales del segundo y tercer molar según la clasificación de Winter: Vertical: -10 a $+10^{\circ}$; Mesioangular: 11 a $79^{\circ}$;
Horizontal: 80 a $100^{\circ}$; Distoangular: -11 a $-79^{\circ}$; Bucolingual: Superposición de corona y raíces y Otros: 111 a $-80^{(1)}$.

Los resultados se registraron en una hoja de cálculo de Microsoft Office Excel. Para el análisis estadístico se empleó el programa SPSS vs.26 (IBM, EE.UU.). Donde los análisis se realizaron de acuerdo a los objetivos planteados para esta investigación. Se fijó un nivel de significancia del $5 \%$ con un intervalo de confianza del 95\%. En la estadística descriptiva fueron representados por tablas de frecuencia y porcentaje.

El presente estudio tomó en cuenta los principios de la Declaración de Helsinki, los valores éticos como el respeto y la confidencialidad de los datos evaluados ${ }^{(12)}$. Asimismo, el estudio fue aprobado por el Comité de Investigación de la Universidad César Vallejo.

\section{RESULTADOS}

Se observó que la posición de los terceros molares según la clasificación de Winter, mesioangular y vertical fueron las más frecuentes. La posición vertical se registró con mayor frecuencia en la pieza 48 con un 14.6\%; mientras que, la posición mesioangular se registró con mayor frecuencia en la pieza 38 con un $14.4 \%$. Asimismo, se observó que la posición vertical se presentó en la pieza 38 con un $13.2 \%$ y la posición mesioangular en la pieza 48 con un $12.5 \%$. (Tabla 1). Se encontró que la posición más frecuente de los terceros molares inferiores según tipo, fue $A$ y $B$. La posición de tipo $B$, se registró con mayor frecuencia en la pieza 48 con un $15.4 \%$ y en la pieza 38 con un $14.4 \%$; mientras que, la posición tipo $\mathrm{A}$ se registró en el $13.8 \%$ de la pieza 38 y el $12.5 \%$ de la pieza 48 . El $29.8 \%$ de las terceras molares inferiores tuvieron una posición tipo $B$, seguido de un 26.3\% con una posición tipo $A$, según Pell y Gregory. (Tabla 2). Se observó que la posición más frecuente de los terceros molares según clase, fueron I y II. La posición clase II, se registró con mayor frecuencia en el $18.8 \%$ de la pieza 48 y el $16 \%$ de la pieza 38 ; mientras que, la posición clase $\mathrm{I}$ se registró con mayor frecuencia en el $12.6 \%$ de la pieza 38 y el $12.1 \%$ de la pieza 48 . El $34.8 \%$ de las terceras molares inferiores tuvieron una posición clase II yel $24.7 \%$ una posición clase I, según Pell y Gregory. (Tabla 3). 
Tabla 1. Posición de los terceros molares inferiores según la clasificación de Winter en pacientes atendidos en un centro de diagnóstico por imágenes. Lima, 2017-2020.

\begin{tabular}{|c|c|c|c|c|c|c|c|c|}
\hline & & & BUCOLINGUAL & DISTOANGULAR & HORIZONTAL & MESIOANGULAR & NR & VERTICAL \\
\hline $\begin{array}{c}\mathrm{N}^{\circ} \text { de } \\
\text { Tercera } \\
\text { molar }\end{array}$ & & Recuento & 1 & 11 & 37 & 73 & 64 & 67 \\
\hline \multirow[t]{3}{*}{ inferior Pza. } & 38 & $\%$ del total & $0.2 \%$ & $2.2 \%$ & $7.3 \%$ & $14.4 \%$ & $12.6 \%$ & $13.2 \%$ \\
\hline & & Recuento & 3 & 18 & 33 & 63 & 62 & 74 \\
\hline & 48 & $\%$ del total & $0.6 \%$ & $3.6 \%$ & $6.5 \%$ & $12.5 \%$ & $12.3 \%$ & $14.6 \%$ \\
\hline \multirow[t]{2}{*}{ Total } & & Recuento & 4 & 29 & 70 & 136 & 126 & 141 \\
\hline & & $\%$ del total & $0.8 \%$ & $5.7 \%$ & $13.8 \%$ & $26.9 \%$ & $24.9 \%$ & $27.9 \%$ \\
\hline
\end{tabular}

Fuente: Elaboración propia (2021).

Tabla 2. Posición de los terceros molares inferiores según tipo de la clasificación de Pell y Gregory, en pacientes atendidos en un centro de diagnóstico por imágenes. Lima, 2017-2020

\begin{tabular}{|c|c|c|c|c|c|c|}
\hline & & & \multicolumn{4}{|c|}{ Clasificación de Pell Gregory (Tipo) } \\
\hline & & & $A$ & B & $\mathrm{C}$ & NR \\
\hline \multirow{4}{*}{$\mathrm{N}^{\circ}$ de Tercera Molar Inferior. } & \multirow{2}{*}{38} & Recuento & 70 & 73 & 46 & 63 \\
\hline & & $\%$ del total & $13.8 \%$ & $14.4 \%$ & $9.1 \%$ & $12.5 \%$ \\
\hline & \multirow{2}{*}{48} & Recuento & 63 & 78 & 50 & 62 \\
\hline & & $\%$ del total & $12.5 \%$ & $15.4 \%$ & $9.9 \%$ & $12.3 \%$ \\
\hline \multirow{2}{*}{ Total } & & Recuento & 133 & 151 & 96 & 125 \\
\hline & & $\%$ del total & $26.3 \%$ & $29.8 \%$ & $19.0 \%$ & $24.7 \%$ \\
\hline
\end{tabular}

Tabla 3. Posición de los terceros molares inferiores según clase de la clasificación de Pell y Gregory, en pacientes atendidos en un centro de diagnóstico por imágenes. Lima, 2017-2020.

\begin{tabular}{|c|c|c|c|c|c|c|}
\hline & & & \multicolumn{4}{|c|}{ Clasificación de Pell Gregory (Clase) } \\
\hline & & & I & II & III & NR \\
\hline \multirow{4}{*}{$\mathrm{N}^{\circ}$ de Tercera Molar Inferior } & \multirow{2}{*}{38} & Recuento & 64 & 81 & 44 & 63 \\
\hline & & $\%$ del total & $12.6 \%$ & $16.0 \%$ & $8.7 \%$ & $12.5 \%$ \\
\hline & \multirow{2}{*}{48} & Recuento & 61 & 95 & 35 & 62 \\
\hline & & $\%$ del total & $12.1 \%$ & $18.8 \%$ & $6.9 \%$ & $12.3 \%$ \\
\hline \multirow{2}{*}{ Total } & & Recuento & 125 & 176 & 79 & 125 \\
\hline & & $\%$ del total & $24.7 \%$ & $34.8 \%$ & $15.6 \%$ & $24.7 \%$ \\
\hline
\end{tabular}

Fuente: Elaboración propia (2021). 


\section{DISCUSIÓN}

Se recolectó la información de una base de datos, la cual estuvo conformada por 253 radiografías panorámicas de pacientes. Se utilizó para el presente estudio la clasificación de Winter y Pell \& Gregory, para poder determinar la posición, tipo y clase de los terceros molares inferiores, donde se toma en cuenta el espacio que existe entre el borde anterior de la rama ascendente de la mandíbula y la cara distal del segundo molar, y para el tipo toma la profundidad del tercero molar en relación a la segunda molar; mientras que, en la clasificación de Winter toma en cuenta la posición del tercero molar en relación con el eje axial del segundo molar $(2,10,16,4,3,11)$.Dando como resultado de acuerdo a las clasificaciones mencionadas, que hubo un posición de tercera molar inferior impactado en el $37.7 \%$ para la pieza 38 y en $37.8 \%$ para la pieza 48 . Asimismo, las posiciones mesioangular y vertical según la clasificación de Winter; así como, la posición nivel B y clase II según Pell y Gregory fueron los más frecuentes.

Dentro de las características sociodemográficas, se encontró que la presencia de terceros molares fue mayor en el grupo de 18 a 30 años; así como, en el sexo femenino; siendo similar al estudio realizado por Gupta $^{8}$, et al. 2011 quienes evaluaron 988 terceros molares mandibulares impactados, en 578 personas, de 18 años a más, clasificándolas según edad, sexo y lado mandibular. De 578 personas, 307 eran hombres en un $53,11 \%$ y 271 mujeres al $46,89 \%$. Siendo el número máximo de terceros molares impactadas, al grupo de edad de 18 a 27 años, en un 68,89\%. Encontrando una diferencia estadística según género de acuerdo con el presente estudio, pero una asemejanza con respecto a la edad presentada y a la similitud de objetivos obtenidos. Asimismo, en comparación con Nagaraj ${ }^{(11)}$, et al. 2016, quienes realizaron un estudio en pacientes con el grupo de edad de 18 a 30 años en la India, fueron interpretados y evaluados estadísticamente dando como resultado, que según el sexo; corresponde un $58,2 \%$ en varones y $41,8 \%$ en mujeres. A diferencia del presente estudio, que compara con el rango de edad; sin embargo, el porcentaje si fue mayor en los varones.

Con respecto al lado mandibular, la presencia de terceros molares fue aproximadamente del $50 \%$ tanto para el lado derecho como izquierdo. Al igual que en nuestro estudio, los autores anteriores (1-5,8) notaron que la posición del tercer molar mandibular era bilateral en la mayoría de los casos; con una posición impactada de aproximadamente el $37 \%$ en ambos lados. Sin embargo, a diferencia de nuestro estudio, se observó la mayor frecuencia de impactación en el lado izquierdo. Parece que la frecuencia de la impactación bilateral, del lado derecho y del lado izquierdo se ve afectada por factores como la genética y la raza $^{(3,5,7)}$. Según la clasificación de Winter, se encontró que la posición, mesioangular $y$ vertical, fue con mayor frecuencia en las piezas 38 y 48.

En el caso vertical se obtuvo $14.6 \%$ y $13.2 \%$ para dichas piezas molares, mientras que, para la posición mesioangular un $12.5 \%$ y $14.4 \%$.este resultado coincide con lo reportado por Demirel (26), et al. 2019.Se encontró que 90 terceros molares evaluados impactados, su posición más frecuente fue mesioangular con un $34,4 \%$, seguida de vertical, horizontal y distoangular, siendo su objetivo evaluar la posición de impactación del tercer molar y el ángulo gonial utilizando como clasificación a Winter, teniendo un resultado parecido a esta investigación presente. Mientras que Da Silva. ${ }^{7}$, et al. 2018, realizó un estudio que comprendió 1205 terceros molares mandibulares en 710 radiografías panorámicas evaluadas en Brasil, y se evaluó con respecto a la posición, según la clasificación de Winter, dando un resultado al 53,8\%, donde se demostró que el tipo de tercer molar mandibular más prevalente en pacientes brasileños fue la posición mesioangular.

Así mismo, comparando con esta literatura se fundamenta lo encontrado en esta investigación. Concluyendo que, la posición más común fue mesioangular y vertical en el lado derecho; y vertical y mesioangular en el lado izquierdo. Una vez más la literatura muestra que el ángulo más común es vertical y mesioangular, en un estudio desarrollado en India ${ }^{(13)}$ y vertical en un estudio realizado en Turquía. ${ }^{1}$ En la mayoría de los estudios previos, la posición mesioangular fue el tipo más común ${ }^{(1-9)}$. Estudios previos han demostrado que la posición mesioangular se asocia con un mayor riesgo de caries en la superficie distal de los segundos molares mandibulares ${ }^{(1,3,5)}$. 
En tales casos, la extracción de los terceros molares mandibulares debe realizarse para prevenir caries en la superficie distal de una zona adyacente del segundo diente molar. Ryalat ${ }^{(10)}$, et al. en el 2018 y Kazemian $^{(2)}$, et al. en el 2015, encontraron una mayor frecuencia de posición vertical, similar, a lo reportado en este estudio.

En cuanto a la clasificación de Pell y Gregory, según su tipo, se observó que en las piezas molares 38 y 48, las posiciones más frecuentes son $A$ y $B$. En el caso de la posición $A$ se tiene $13.8 \%$ y $12.5 \%$ para dichas piezas molares; mientras que, para la posición B se tiene un $15.4 \%$ y $14.4 \%$ respectivamente. A diferencia de,

$\operatorname{Rezaei}^{(1)}$, et al. 2020, que encontró con mayor frecuencia un nivel $C$ en un $40,3 \%$ y clase I al $63,7 \%$. Este estudio mostró que el nivel B fue la profundidad de impactación más común tanto en el lado derecho $15,4 \%$ e izquierdo $14,4 \%$, lo que está de acuerdo con los resultados observados. Según su clase, se demostró en el presente estudio que en las piezas molares 38 y 48 según la clasificación de Pell \& Gregory se encuentran con mayor frecuencia la posición clase I y II, donde se puede observar que estas categorías tienen los mayores porcentajes.

En el caso de la posición clase I se tiene $12.6 \%$ y $12.1 \%$ para dichas piezas molares, mientras que, para la posición clase II se tiene el valor más alto con un $16.0 \%$ y $18.8 \%$ respectivamente, este resultado coincide que la posición más común de los terceros molares impactados en relación con la rama mandibular fue de Clase II en ambos lados, corroborando hallazgos previos (1-6). Comparando con el autor Yunus $\left.{ }^{(19}\right)$, et al. 2020, obtuvo como resultado un porcentaje total $32,9 \%$,en la clase I ,siendo de mayor frecuencia en este estudio la clase teniendo una similitud con el presente estudio. Dentro de las limitaciones de este estudio, está el tipo de diseño, de naturaleza retrospectiva y el tamaño muestral evaluado; recomendando aumentar la potencia estadística de la prueba en futuros estudios. Sin embargo, la información dada, sirve de aporte a los cirujanos dentistas o especialistas; ya que brinda información de utilidad respecto a la posición de terceros molares para evitar complicaciones en el paciente durante el acto quirúrgico. Una de las fortalezas de esta investigación, es que se utilizan dos clasificaciones para obtener valores más precisos a diferencia de estudios previos realizados enfocados en un solo tipo de clasificación.
Se concluye que, la posición de impactación de los terceros molares inferiores más frecuente según la clasificación de Winter fue la posición vertical; seguida de la posición mesioangular; y según la clasificación de Pell y Gregory, fueron el tipo B y clase II. Es importante conocer las diferentes posiciones, tipos y clases de terceros molares inferiores debido a sus variaciones anatómicas; por ello, es necesario evaluar sus características radiográficas para realizar un buen diagnóstico y garantizar un tratamiento quirúrgico adecuado para evitar complicaciones futuras en la práctica profesional

Contribuciones de autoría: SLPU, SCMK y MKSC diseñaron el estudio, recolectaron los datos y realizaron el procesamiento y análisis estadístico. Las autoras redactaron el manuscrito y aprobaron la versión final del mismo.

Fuente de financiamiento: El estudio fue financiado por las autoras.

Conflicto de intereses: Las autoras declararon no tener conflictos de interés.

\section{REFERENCIAS BIBLIOGRAFICAS}

1. Rezaei F, Imani MM, Khavid A, Nabavi A. Patterns of Mandibular Third Molar Impaction in an Iranian Subpopulation. Pesqui Bras Odontopediatria Clin Integr [Internet]. 2020;20:1. Available http://www.scielo.br/scielo.php?script=sci_arttext \&pid=S1983-46322020000100388\&tIng=en

2. Kazemian M, Zarch S, Banihashemi E, Khajavi M, Moradi E. Frequency of Impacted Teeth in Patients Referred to a Radiology Center and the Radiology Depart- ment of Mashhad School of Dentistry. Bangladesh J Med Sci Vol [Internet]. 2015;14(2):165-8. Available from: http://journals.sagepub.com/doi/10.1177/146144 4810365020

3. Muhammad S, Hasan U, Shafiq F, Rasheed A, Owais S, Jaffary SB. Radiographic Evaluation of Impacted Mandibular Third Molars: a CrossSectional Study. Pakistan Oral Dent J [Internet]. 2017;37(1):26. Available from: http://podj.com.pk/archive/March_2017/PODJ6.pdf

4. Santosh P. Impacted mandibular third molars: Review of literature and a proposal of a combined clinical and radiological classification. Ann Med Health Sci Res [Internet]. 
2015;5(4):229. Available from: http://www.amhsr.org/text.asp?2015/5/4/229/16017 7

5. Khouri C, Aoun G, Khouri C, Saade M, Salameh Z, Berberi A. Evaluation of Third Molar Impaction Distribution and Patterns in a Sample of Lebanese Population. J Maxillofac Oral Surg [Internet]. 2020 Jul 16; Available from: https://doi.org/10.1007/s12663-020-01415-x

6. Goyal S, Verma P, Sunder Raj S. Radiographic Evaluation of the Status of Third Molars in Sriganganagar Population - A Digital Panoramic Study. Malaysian J Med Sci [Internet]. 2016;23(6):103-12. Available from: http://journal.usm.my/journal/11mjms236_OA8.pdf

7. Da Silva Sampieri MB, Viana FLP, Cardoso CL, Vasconcelos MF, Vasconcelos MHF, Gonçales ES. Radiographic study of mandibular third molars: evaluation of the position and root anatomy in Brazilian population. Oral Maxillofac Surg [Internet]. 2018 Jun 15;22(2):163-8. Available from: http://link.springer.com/10.1007/s10006-018-0685y

8. Gupta S, Bhowate RR, Nigam N, Saxena S. Evaluation of Impacted Mandibular Third Molars by Panoramic Radiography. ISRN Dent [Internet]. 2011 Sep 29;2011(M):1-8. Available from: https://www.hindawi.com/journals/isrn/2011/406714 I

9. Prajapati V, Mitra R VK. Pattern mandibular third molar impaction its Assoc to caries mandibular Second molar A Clin Var. Dent Res J (Isfahan). 2017;14(2):137-142.

10. Machado De Lima DV. Research Design: a contribution To the Author. Online Brazilian J Nurs [Internet]. 2011;10(2):1-19. Available from: http://www.redalyc.org/articulo.oa?id=36144167401
1

11. Nedel WL, Silveira F da. Different research designs and their characteristics in intensive care. Rev Bras Ter Intensiva [Internet]. 2016;28(3):256-60. Available from: http://www.gnresearch.org/doi/10.5935/0103507X.20160050

12. Association world medical. Declaration of Helsinki: ethical principles for medical research involving human subjects. Clin Rev Spec [Internet]. 2013;310(20):2013-6. Available from: https://jamanetwork.com/journals/jama/fullarticle/17 60318

13. Ryalat S, AIRyalat SA, Kassob Z, Hassona Y, AlShayyab $\mathrm{MH}$, Sawair F. Impaction of lower third molars and their association with age: radiological perspectives. BMC Oral Health [Internet]. 2018 Dec 4;18(1):58. Available from: https://bmcoralhealth.biomedcentral.com/articles/1 0.1186/s12903-018-0519-1

14. Nagaraj T, Balraj L, Irugu K, Rajashekarmurthy $S$, Sreelakshmi. Radiographic assessment of distribution of mandibular third molar impaction: A retrospective study. J Indian Acad Oral Med Radiol [Internet]. 2016;28(2):145. Available from: http://www.jiaomr.in/text.asp?2016/28/2/145/19512 5

15. Demirel O, Akbulut A. Evaluation of the relationship between gonial angle and impacted mandibular third molar teeth. Anat Sci Int [Internet]. 2020 Jan 7;95(1):134-42. Available from: https://doi.org/10.1007/s12565-019-00507-0

16. Yunus B, Wulansari DP, Tunnizha BM. Lower third molar impaction based on pell and gregory classification: A panoramic study. Syst Rev Pharm. 2020;11(11):19-23.

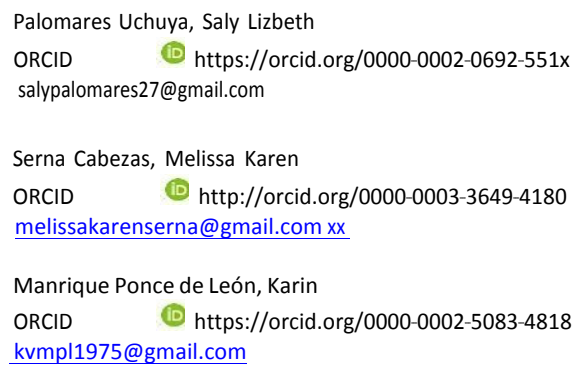

Copyright $\odot$ La revista. La revista Kiru es publicada por la Facultad de Odontología de la Universidad de San Martín de Porres, en Lima, Perú. 